\section{Three-dimensional higher-order Padé approximant-based wide-angle beam propagation method using complex Jacobi iteration}

Khai, Q., Le and P. Bienstman

Wide-angle scalar beam propagation methods (BPMs) for effective modelling of optical propagation in three-dimensional (3D) waveguides are usually limited to the low-order-accurate Padé $(1,1)$ approximant. Presented is a 3D wide-angle scalar BPM based on higher-order Padé approximant operators which is factored into a series of simpler first-order Padé(1,1) approximants. This results in a multistep method where each step can be cast in terms of a 2D Helmholtz equation with source term, which can be treated accurately and effectively by the new complex Jacobi iterative technique.

Introduction: Algorithms for accurate modelling of optical propagation in $3 \mathrm{D}$ waveguide structures are critical for the design of photonics devices. One of the most widely used techniques for the study of 3D optical waveguide devices that vary slowly in the propagation direction is an alternating-direction implicit (ADI) finite difference (FD) beam propagation method (BPM) [1]. However, this method is only firstorder-accurate in the propagation step size and higher-order methods are thus required for acceptable accuracy if wide-angle (WA) or nonparaxial propagation is needed. Efforts have been made to relax the limitations for WA simulations. A 3D multistep horizontal WA-BPM based on the generalised Douglas scheme, together with an ADI scheme was developed in [2]. However, the WA propagator is only a Padé $(1,1)$ approximant operator. Recently, Ma and Keuren [3] presented a new 3D WA-BPM based on Hoekstra's scheme, which does not require the splitting of the Fresnel wave equation or use of an ADI method. By using a technique to shift the simulation window to reduce the dimension of the numerical equation and a threshold technique to ensure further its convergence, this approach shows accuracy and effectiveness and also enables one to develop a higher-order Padé approximant-based WA-BPM. However, the resultant propagation scheme can be very slow if either the problem size is large or the structure or boundary conditions are changing as the propagation proceeds, requiring frequent reinversions of the matrix. To circumvent this problem, we previously presented an effective 3D WA-BPM using the new complex Jacobi iterative (CJI) technique that is very well-suited for large problems [4]. However, this technique is also only based on a low-order Padé(1,1) approximant.

Several years ago, a 2D WA-BPM based on a higher-order Padé(3,3) approximant operator was reported. The beam propagation equation is expressed as factors of the first-order Padé $(1,1)$ approximant-based equation, thus leading to the simplified multistep algorithm [5]. Since each component step could be described in a tridiagonal matrix form, it is usually solved by the well-known direct matrix inversion (DMI). However, the method was originally limited to $2 \mathrm{D}$ structures owing to the low effectiveness of DMI. In this Letter, we overcome this problem by presenting a 3D multistep WA scalar BPM based on a higher-order Padé $(3,3)$ approximant operator where each 3D component step is cast in terms of a 2D Helmholtz equation with source term, which can be effectively solved by the new CJI method. The effectiveness of this iterative technique for WA-BPM is demonstrated in comparison with the traditional DMI method.

Formulations: Modified Padé approximant operators for WA-BPM: The scalar Helmholtz equation obtained using the slowly varying envelope approximation is given by [4]

$$
\frac{\partial H}{\partial z}-\frac{i}{2 k} \frac{\partial^{2} H}{\partial z^{2}}=\frac{i P}{2 k} H
$$

where $P=\nabla_{\perp}^{2}+k_{0}^{2}\left(n^{2}-n_{r e f}^{2}\right)=\left(\partial^{2} / \partial x^{2}\right)+\left(\partial^{2} / \partial y^{2}\right)+k_{0}^{2}\left(n^{2}-n_{r e f}^{2}\right)$ with $k=k_{0} n_{r e f}, n$ is the refractive index profile, $n_{\text {ref }}$ the reference refractive index, and $k_{0}$ is the vacuum wavevector. Hadley [6] proposed the rational approximation of WA beam propagation using the following recurrence relation:

$$
\left.\frac{\partial}{\partial z}\right|_{n+1}=i \frac{P / 2 k}{1-(i / 2 k) \partial /\left.\partial z\right|_{n}}
$$

For initial value of $\partial /\left.\partial z\right|_{0}=0$, this gives us the well-known Padé $(m, n)$ approximant-based WA beam propagation formula as follows:

$$
\frac{\partial H}{\partial z} \simeq i \frac{N(m)}{D(n)} H
$$

where $N(m)$ and $D(n)$ are polynomials in $P$. If (3) is compared with a formal solution of (1) written in the well-known form

$$
\frac{\partial H}{\partial z}=i\left(\sqrt{P+k^{2}}-k\right) H=i k(\sqrt{1+X}-1) H
$$

where $X=P / k^{2}$, we obtain the approximation formula

$$
\sqrt{1+X}-1 \simeq \frac{N(m)}{D(n)}
$$

However, we recently presented a modified Padé approximant of the WA propagator by using the same recurrence relation (2) with a different initial value of $\partial /\left.\partial z\right|_{0}=-k \beta$ ( $\beta>0$ is a damping parameter). The resulting approach not only allows a more accurate approximation to the true Helmholtz equation than the $\operatorname{Hadley}(m, n)$ approximant but also gives evanescent modes the desired damping as shown in Figs. 1 and 2, respectively. It is obvious that the higher the Padé approximant is, the more accurate is the approximation to the true Helmholtz equation

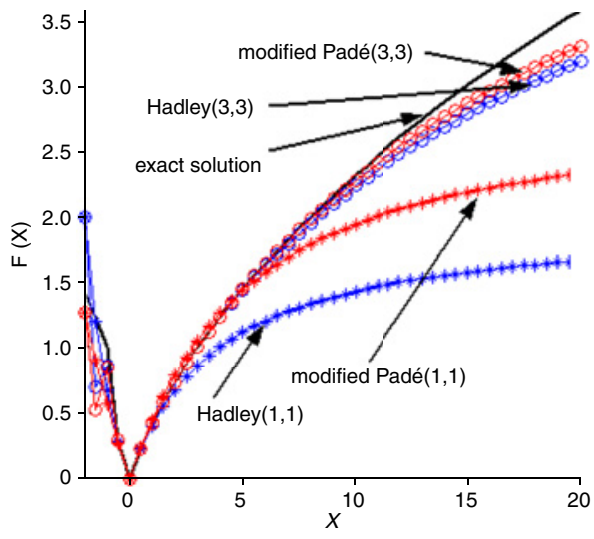

Fig. 1 Absolute values of $(1+X) 1 / 2-1$ (solid line), standard and modified Padé $(1,1)$ and Padé $(3,3)$ approximant of $(1+X) 1 / 2-1$ with respect to $X$

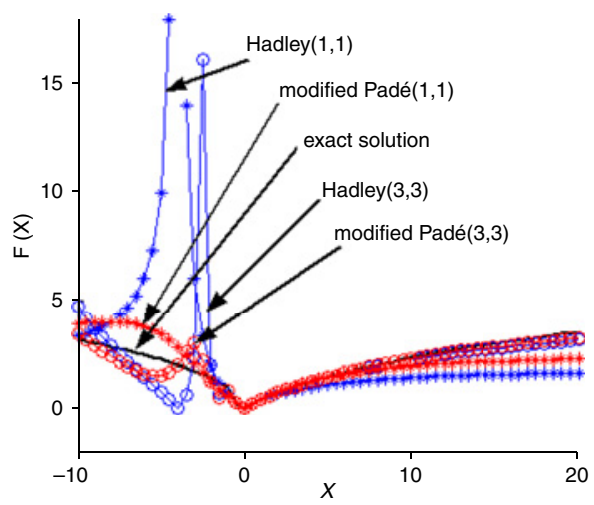

Fig. 2 Absolute values of $(1+X) 1 / 2-1$ (solid line), standard and modified Padé $(1,1)$ and Padé $(3,3)$ approximant of $(1+X) 1 / 2-1$ with respect to $X$

$3 D$ multistep method for WA-BPM: By using the standard Padé $(N, N)$ approximant operator, the scalar Helmholtz equation may be decomposed into an $N$-step algorithm for which the $k$ th partial step takes the form [5]

$$
\phi^{n+k / N}=\frac{1+a_{k}^{*} P}{1+a_{k} P} \phi^{n+(k-1) / N}
$$

$a_{k}^{*}$ is the complex conjugate of $a_{k}$. This form also holds for those based on our modified $\operatorname{Padé}(N, N)$ approximant operator, but with different 
coefficients $a_{k}$ and $a_{k}^{*}$. For example, with $N=2$, we obtain

$$
\begin{aligned}
a_{1}+a_{2} & =\left(2+\frac{1}{1+i \beta / 2}\right) \frac{1}{4 k^{2}}-\frac{i \Delta z}{4 k} \\
a_{1} a_{2} & =\frac{1}{16 k^{4}(1+i \beta / 2)}-\frac{i \Delta z}{16 k^{3}}\left(1+\frac{1}{1+i \beta / 2}\right) \\
a_{1}^{*}+a_{2}^{*} & =\left(2+\frac{1}{1+i \beta / 2}\right) \frac{1}{4 k^{2}}+\frac{i \Delta z}{4 k} \\
a_{1}^{*} a_{2}^{*} & =\frac{1}{16 k^{4}(1+i \beta / 2)}+\frac{i \Delta z}{16 k^{3}}\left(1+\frac{1}{1+i \beta / 2}\right)
\end{aligned}
$$

It is obvious that the modified Padé approximant reduces to the standard Padé approximant when we set $\beta=0$. From (6), it is clearly seen that each partial step is a simple Padé $(1,1)$ approximant-based 3D WA beam propagation equation. For beam propagation of wave profiles of a $2 \mathrm{D}$ cross-section, each $3 \mathrm{D}$ propagation equation can be recast in terms of a 2D Helmholtz equation with source term and the effective absorption coefficients appearing in these equations are relatively high. This can lead to rapid convergence of the new complex Jacobi method. For more details on this approach, we refer to [4].

Benchmark results: That higher-order Padé(3,3) approximants yield more accurate results was already shown in [6]. There, however, 3D methods were not practical in view of the slowness of DMI. To show the effectiveness of the higher-order Padé $(3,3)$ approximant-based WA-BPM using the new CJI method compared with those of traditional DMI, we performed several benchmarks on 3D optical waveguide structures. The beam propagation equation based on the Padé(3,3) approximant is expressed as factors using Padé $(1,1)$ approximants. Then each is recast in terms of a 2D inhomogenous Helmholtz equation. The simulated examples are Gaussian beam propagation in a 3D straight rib waveguide and guided-mode propagation in a Y-branch rib waveguide [4] and are all run on a notebook PC using Matlab.

The width and height of the straight rib waveguide are $w=2 \mu \mathrm{m}$ and $h=1.1 \mu \mathrm{m}$, as in Fig. 4 of [7]. The guiding core has an index $n f=3.44$ and a thickness $t=0.2 \mu \mathrm{m}$ while the refractive index of the substrate and of the cover is $n s=3.4$ and $n c=1$, respectively. The Gaussian beam with a waist radius $w 0=0.3 \mu \mathrm{m}$ has been injected into the rib waveguide at wavelength $\lambda=1.55 \mu \mathrm{m}$. Owing to the large memory required for DMI, the small computational window of $2 \times 2 \mu \mathrm{m}$ is discretised with a grid size of $\Delta x=\Delta y=0.1 \mu \mathrm{m}$, and the short path length of $2 \mu \mathrm{m}$ is discretised with a propagation step size $\Delta z=0.1 \mu \mathrm{m}$.

For a Y-branch, the initial rib waveguide is split into two $5^{\circ}$ tilted waveguides. The longitudinal dimension is $h 1=1 \mu \mathrm{m}$. The other structure parameters are the same as the above straight rib waveguide. The fundamental TE mode of the ridge waveguide of width $w=2 \mu \mathrm{m}$ at $1.55 \mu \mathrm{m}$ wavelength is used as the excited field at $z=0$. The propagation step size is $\Delta z=0.1 \mu \mathrm{m}$.

The resulting runtimes of the 3D WA-BPM using the DMI and the CJI method are listed in Table 1. It is clearly shown that the runtime of the iterative method is substantially lower than that of the DMI method. For large problems requiring very large storage space and also for structures with a long path length with a small propagation step size that require frequent matrix inversions, the DMI technique is numerically very intensive. In contrast, for typical choices of $\mathrm{k} \Delta \mathrm{z}$ the CJI technique offers rapid convergence and shorter runtimes.

Table 1: Quantitative comparision of runtimes of direct matrix inversion and complex Jacobi iteration for WA-beam propagation in waveguide (WG) structures

\begin{tabular}{|c|c|c|}
\hline Structure & \multicolumn{2}{|c|}{$3 \mathrm{D}$} \\
\hline Method & Straight rib WG & Y-branch rib WG \\
\hline DMI & $550.2 \mathrm{~s}$ & $769.7 \mathrm{~s}$ \\
\hline CJI & $35.1 \mathrm{~s}$ & $100.8 \mathrm{~s}$ \\
\hline
\end{tabular}

Conclusion: A 3D WA-BPM based on higher-order modified Padé approximant operators using the new complex Jacobi iteration has been developed. The solution technique results in higher accuracy than our previous approach. The excellent effectiveness of this new iterative method in terms of shorter runtime has been demonstrated compared with those of the traditional direct matrix inversion.

Acknowledgment: Parts of this work were performed within the context of the Belgian IAP project Photonics@Be.

(C) The Institution of Engineering and Technology 2010

13 November 2009

doi: $10.1049 / \mathrm{el} .2010 .3204$

One or more of the Figures in this Letter are available in colour online.

Khai, Q., Le and P. Bienstman (Department of Information Technology, Ghent University-IMEC, St-Pietersnieuwstraat 41, Ghent B-9000, Belgium)

E-mail: khai.le@intec.ugent.be

\section{References}

1 Kawano, K., and Kitoh, T.: 'Introduction to optical waveguide analysis solving Maxwell's equation and Schrödinger's equation' (Wiley, New York, 2001), pp. 251-254

2 Shibayama, J., Takahashi, T., Yamauchi, J., and Nakano, H.: 'A threedimensional multistep horizontally wide-angle beam-propagation method based on the generalized Douglas scheme', IEEE Photonics Technol. Lett., 2006, 18, (23), pp. 2535-2537

$3 \mathrm{Ma}, \mathrm{C}$., and Keuren, E.V.: 'A three-dimensional wide-angle beam propagation method for optical waveguide structures', Opt. Express, 2007, 15, (2), pp. 402-407

4 Le, Khai, Q., Godoy-Rubio, R., Bienstman, P., and Hadley, G.R.: 'The complex Jacobi iterative method for three-dimensional wide-angle beam propagation', Opt. Express, 2008, 16, (21), pp. 17021-17030

5 Hadley, G.R.: 'Multistep method for wide-angle beam propagation', Opt. Lett., 1992, 17, (24), pp. 1743-1745

6 Hadley, G.R.: 'Wide-angle beam propagation using Padé approximant operators', Opt. Lett., 1992, 17, (20), pp. 1426-1428

7 Tsuji, Y., Koshiba, M., and Shiraishi, T.: 'Finite element beam propagation method for three-dimensional optical waveguide structures', J. Lightwave Technol., 1997, 15, (9), pp. 1728-1734 